Article

\title{
A Dual-Polymer Fiber Fizeau Interferometer for Simultaneous Measurement of Relative Humidity and Temperature
}

\author{
Chao-Tsung Ma ${ }^{1}$, Yu-Wei Chang ${ }^{2}$, Yuan-Jie Yang ${ }^{2}$ and Cheng-Ling Lee ${ }^{2, *}$ \\ 1 Department of Electrical Engineering, National United University, Miaoli 36003, Taiwan; ctma@nuu.edu.tw \\ 2 Department of Electro-Optical Engineering, National United University, Miaoli 36003, Taiwan; \\ as85635544@gmail.com (Y.-W.C.); nn4088nn40888@gmail.com (Y.-J.Y.) \\ * Correspondence: cherry@nuu.edu.tw; Tel.: +886-37-382568; Fax: +886-37-382555
}

Received: 19 October 2017; Accepted: 16 November 2017; Published: 17 November 2017

\begin{abstract}
This paper presents a novel design method in which a dual-polymer fiber Fizeau interferometer (DPFFI) is proposed for simultaneously measuring relative humidity (RH) and temperature $(\mathrm{T})$. Since the polymer is intrinsically highly sensitive to both $\mathrm{RH}$ and $\mathrm{T}$, the polymer fiber Fizeau interferometer (PFFI) exhibits cross-sensitivity of RH and T. In general, it is difficult to demodulate the optical responses from both variations of RH and T using a single PFFI. If two PFFIs with different structures are combined, they will individually exhibit distinct sensitivity responses with respect to RH and T, respectively. The technical problem of analyzing multiple interferences of the optical spectra of the DPFFI and the individual sensitivity of RH and T to each PFFI is obtained using the fast Fourier transform (FFT). A mathematical method is applied to solve the simultaneous equations of the DPFFI, so that the two variables $\mathrm{RH}$ and $\mathrm{T}$ can be determined at the same time. Experimental results, indicating good sensitivity and accuracy, with small measurement errors (average errors of $\sim 1.46^{\circ} \mathrm{C}$ and $\sim 1.48 \%$, respectively), are shown, determining the feasibility, and verifying the effectiveness, of the proposed DPFFI sensor.
\end{abstract}

Keywords: Fiber Fizeau interferometer (FFPI); polymer; fiber sensor; fast Fourier transform (FFT); simultaneously sensing humidity and temperature

\section{Introduction}

Relative humidity (RH) and temperature ( $\mathrm{T}$ ) are two significant physical parameters affecting a variety of industrial processes, such as semiconductor technology, food processing, biomedical engineering, weather forecasting, and environmental monitoring and control. In the above mentioned processes, the simultaneous measurement of $\mathrm{RH}$ and $\mathrm{T}$ provides a number of engineering advantages and is often a necessity for a variety of system applications. In recent years, several fiber optical sensors for simultaneous $\mathrm{RH}$ and $\mathrm{T}$ sensing have been proposed and investigated, but most of them are incorporated with well-known fiber devices such as fiber Bragg gratings (FBGs) [1-8], long-period fiber gratings (LPFGs) [8-10], and Fabry-Perot interferometers (FPIs) [1,11-13]. In [1], Arregui et al. proposed a sensor head composed of an FBG and a low-finesse FPI for simultaneous RH and T sensing. An $\mathrm{RH}$ measuring range from $11 \%$ to $97 \% \mathrm{RH}$ and for $\mathrm{T}$ ranging from $10^{\circ} \mathrm{C}$ to $85^{\circ} \mathrm{C}$ are shown. In their design case, the sensor head was connected to a single mode optical fiber network, which yielded a new possibility in multiplexing such sensors. An interesting fiber optic hybrid device composed of an FBG and a reflection-type photonic crystal fiber interferometer (PCFI) infiltrated with RH-sensitive agarose for simultaneous $\mathrm{T}$ and $\mathrm{RH}$ measurement was proposed and demonstrated in [2]. A measured T sensitivity of $9.8 \mathrm{pm} /{ }^{\circ} \mathrm{C}$ and an optical power variation of $7 \mathrm{~dB}$ upon a $75 \% \mathrm{RH}$ change were shown. Another fiber device based on an FBG integrated with a photonic crystal fiber (PCF)-based in-fiber 
Mach-Zehnder interferometer (MZI) was developed in [3], where a short PCF was fusion-spliced between two single-mode fibers and coated with a layer of a polyvinyl alcohol (PVA) material. With the $\mathrm{RH}$ measurement range up to $30-95 \% \mathrm{RH}$, the simultaneous measurement has been achieved with resolutions of $1{ }^{\circ} \mathrm{C}$ and $0.13 \% \mathrm{RH}$ for $\mathrm{T}$ and $\mathrm{RH}$, respectively. A knob-integrated FBG was proposed in [4]. By exciting the cladding modes as well as recoupling the reflected cladding modes back into the leading single mode fiber, the proposed sensor reached an RH sensitivity of up to $1.2 \mathrm{~dB} / \%$ RH within an $\mathrm{RH}$ range of $30-95 \%$ and a $\mathrm{T}$ sensitivity of $8.2 \mathrm{pm} /{ }^{\circ} \mathrm{C}$ in the $\mathrm{T}$ range of $25-60{ }^{\circ} \mathrm{C}$. Massaroni C. et al. proposed a configuration based on an array of FBGs for achieving the simultaneous measurement of both $\mathrm{T}$ and RH of gas in a chamber [5]. Another sensor consisting of a Fabry-Perot cavity formed by two identical FBGs was proposed in [6], where the polyimide material was coated on the FBG and also on the cavity with a different thickness. RH and T sensitivities of $1.92 \mathrm{pm} / \% \mathrm{RH}$ and $8.87 \mathrm{pm} /{ }^{\circ} \mathrm{C}$, respectively, with coating thicknesses of $10 \mu \mathrm{m}$ on the FBG and $15 \mu \mathrm{m}$ on the cavity, were shown. A hygroscopic polymer microcavity fiber Fizeau interferometer (PMFFI) incorporating a fiber Bragg grating (FBG) was proposed in [7] for the simultaneous measurement of RH and T. High sensitivity and accuracy were achieved. A long-period fiber grating (LPG)-based sensing head with an in-line FBG was proposed in [8] for simultaneous measurement of RH and T. Measured resolutions of $1.6 \% \mathrm{RH}$ and $2.5^{\circ} \mathrm{C}$ were achieved within an $\mathrm{RH}$ range from 20 to $50 \%$, while $2.4 \% \mathrm{RH}$ and $0.4{ }^{\circ} \mathrm{C}$ resolutions were achieved within an $\mathrm{RH}$ range from 50 to $80 \%$. A fiber loop mirror sensor with a long period grating (LPG) inscribed in the polarization maintaining fiber (PMF) was proposed in [9]. RH and T sensitivities of $0.1723 \mathrm{~nm} / \% \mathrm{RH}$ and $0.2174 \mathrm{~nm} /{ }^{\circ} \mathrm{C}$ with measurement errors of $2.6 \% \mathrm{RH}$ and $0.2{ }^{\circ} \mathrm{C}$, respectively, were demonstrated. The authors of [10] compared the differences between the $\mathrm{RH}$ and $\mathrm{T}$ sensing capabilities of fully and half-coated LPGs and reported a simple and cost-efficient method of measuring both $\mathrm{T}$ and $\mathrm{RH}$, a possible alternative to cascaded LPGs sensing systems. The main contribution of the proposed dual-wavelength-based sensing method is the simultaneous measurement of $\mathrm{RH}$ and $\mathrm{T}$ using only one LPG. Results showed sensitivities of $63.23 \mathrm{pm} / \% \mathrm{RH}$ and $410.66 \mathrm{pm} /{ }^{\circ} \mathrm{C}$ for the attenuation band corresponding to the coated contribution, and $55.22 \mathrm{pm} / \% \mathrm{RH}$ and $405.09 \mathrm{pm} /{ }^{\circ} \mathrm{C}$ for the attenuation band corresponding to the uncoated grating. In 2014, a dielectric multilayer-based fiber optic sensor with simultaneous RH and T measurement capability was proposed in [11]. Average RH and $\mathrm{T}$ sensitivities of $0.43 \mathrm{~nm} / \% \mathrm{RH}$ and $0.63 \mathrm{~nm} /{ }^{\circ} \mathrm{C}$, respectively, when environmental $\mathrm{RH}$ changes from $1.8 \% \mathrm{RH}$ to $74.7 \% \mathrm{RH}$ and $\mathrm{T}$ changes from 21.4 to $38.8{ }^{\circ} \mathrm{C}$, with high repeatability were shown. In [12], an all-silica miniature fiber-optic sensor based on two cascaded FPIs, formed at the tip of an optical fiber to measure $\mathrm{T}$ and $\mathrm{RH}$, was proposed. The authors of [13] proposed a sensing device using an optical fiber Fabry-Perot interferometer. That sensor was constructed by splicing a short length of PCF to a single-mode fiber and coating an ultrathin PVA film onto the PCF's cleaved surface. However, of all the above-reviewed RH/T sensors, both the sensing sensitivity and resolution may not be high enough for certain high-tech applications. Furthermore, most of these fiber-grating-based sensors usually require complicated laser-written fabrication using expensive equipment.

In this work, simultaneous measurement of RH and T using a very simple and easily fabricated, dual-polymer fiber Fizeau interferometer (DPFFI) is presented. The polymer fiber Fizeau interferometer (PFFI) is based on a highly hygroscopic polymer directly coated on the end faces of two fibers. The presented hygroscopic polymer is highly sensitive to $\mathrm{RH}$ and T compared with previously reported fiber gratings. We strategically combined two PFFIs with different polymer lengths for the simultaneous measurement of RH and T. The key technical difficulty consisted in analyzing multiple interferences of optical spectra of the DPFFI and the individual RH and T sensitivities to each PFFI. By using the fast Fourier transform (FFT) and by solving a strategically simultaneous matrix inverse equation, the RH and $\mathrm{T}$ variables could be determined. Higher sensing sensitivities for $\mathrm{RH}$ and $\mathrm{T}$ were achieved, and no expensive equipment for laser-written fabrication of fiber-grating-based sensors was required. The proposed DPFFI device with design parameters of $\mathrm{L}_{1}=43.92 \mu \mathrm{m}$ and $\mathrm{L}_{2}=12.5 \mu \mathrm{m}$ was practically fabricated and tested under many different $\mathrm{T} / \mathrm{RH}$ conditions. Experimental results 
show that the proposed DPFFI sensor is capable of effectively and simultaneously measuring the surrounding RH and T with good accuracy.

\section{Configuration and Sensing Principle}

The hygroscopic polymer Norland optical adhesive 61 (NOA61) is a photo polymerizable monomer that can be made into a solid polymer by ultraviolet (UV) light curing. We have presented a polymer microcavity fiber Fizeau interferometer incorporating an FBG for simultaneously sensing $\mathrm{RH}$ and T [7]. Here in this design, the FBG is not required; however, two hygroscopic polymer fiber Fizeau interferometers (PFFIs) with different cavities are strategically combined to form the proposed DPFFI sensor for achieving the simultaneous measurement of $\mathrm{T}$ and $\mathrm{RH}$, as shown in Figure 1. Figure 1 shows the layer of the proposed polymer acting as a cavity with two reflective interfaces $R_{1}$ and $R_{2}$, which reflects the optical signals back into the single mode fiber again so that reflection interference is achieved by the fiber Fizeau interferometer. By using monitored translation stages, the thick film of the polymer can be attached to the fiber end face and the UV-curing can be applied to form the first PFFI (sensor $r_{2}$ in Figure 1). The coating and UV-curing steps can be repeated several times to obtain the second PPFI with a longer cavity length (sensor ${ }_{1}$ in Figure 1). The optical characteristics of the coating polymer on SMF varies in response to the RH or T of the surrounding changes, affecting the optical length of the Fizeau cavity as well as the optical phase difference between the two reflected beams. According to the results in [7], this hygroscopic polymer was intrinsically highly sensitive to RH as well as $\mathrm{T}$, and the RH/T sensitivities therein depended on the lengths of the polymer cavity. Thus, by combining two PPFIs with different responses of RH and T, respectively, the proposed sensor was able to individually extract the variations of $\mathrm{RH}$ and $\mathrm{T}$ from the surrounding area. Figure 2 displays the experimental setup for performing the simultaneous measurement. The light signal from a broad band light source propagates with a $2 \times 2$ coupler, reflects off the endfaces of sensor ${ }_{1}$ and sensor 2 , and returns to the coupler again. Finally, the combined spectral response readouts can be directly obtained by an optical spectrum analyzer (OSA).

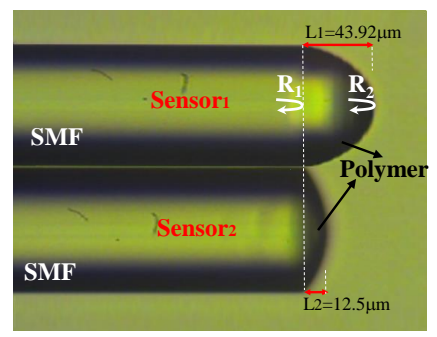

Figure 1. Configuration of the proposed dual-polymer fiber Fizeau interferometer (DPFFI) sensor.

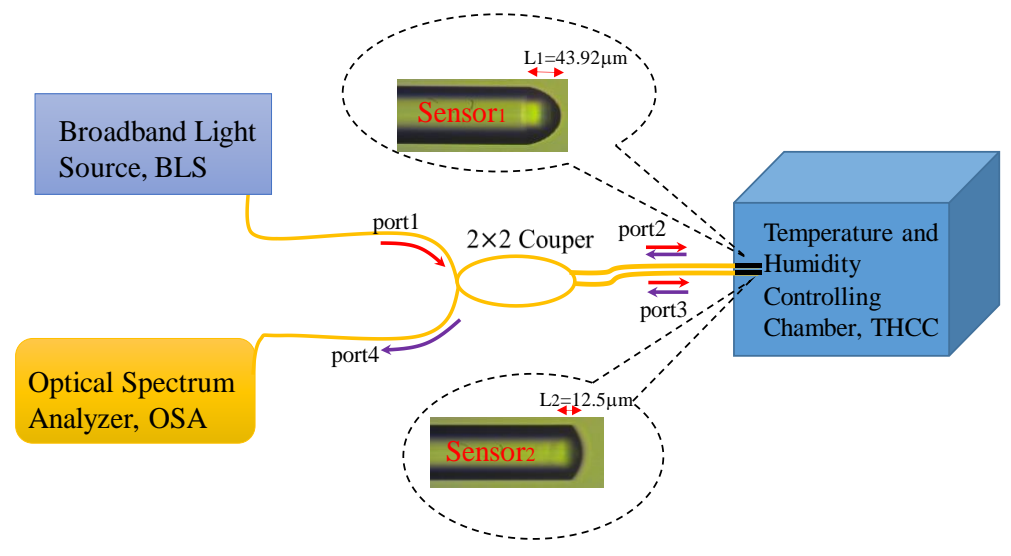

Figure 2. Experimental setup for simultaneously measuring RH and T. 
Since we used two polymer sensors for the measurement of multiple parameters, the optical responses of the DPFFI are superimposed, will be displayed on the OSA. The analysis of the optical responses from the combined interferences can be accomplished using the fast Fourier transform (FFT) method. The FFT method was used to separate multiple interferences in spatial frequency into two individual spatial frequencies for sensor ${ }_{1}$ and sensor 2 , respectively. Figure 3 shows the processes of the optical response of the superimposed interference, separating into the spectra of interference $_{1}$ and interference $e_{2}$ by the FFT method for sensor ${ }_{1}$ and sensor ${ }_{2}$, respectively. Figure $3 a$ shows the multiple interferences of optical responses measured originally by the OSA. The spectral shifts are due to variations in the T and RH of the surrounding area, and the shifts that are caused by $\mathrm{RH}$ and/or T cannot be identified. Therefore, the signals of the superimposed interference of Figure 3a are processed by the FFT to obtain spatial frequency spectra, as shown in Figure $3 \mathrm{~b}$. We can then

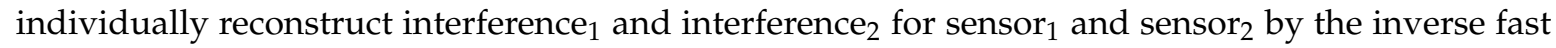
Fourier transform (IFFT), as plotted in Figure 3c,d, respectively. Then, in an identical variation of $T$ or $\mathrm{RH}$, wavelength shifts of the optical interference ${ }_{1}$ and interference for sensor $_{1}$ and sensor ${ }_{2}$ can be independently determined for achieving the simultaneous measurement of $\mathrm{RH}$ and $\mathrm{T}$. In Figure 3c,

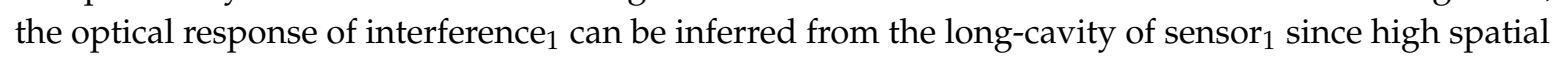
frequency comes from the long-cavity in sensor ${ }_{1}$. It can also be predicted that the sensitivities of RH and $\mathrm{T}$ in the long-cavity sensor ${ }_{1}$ are smaller than those obtained by the short-cavity sensor 2 .

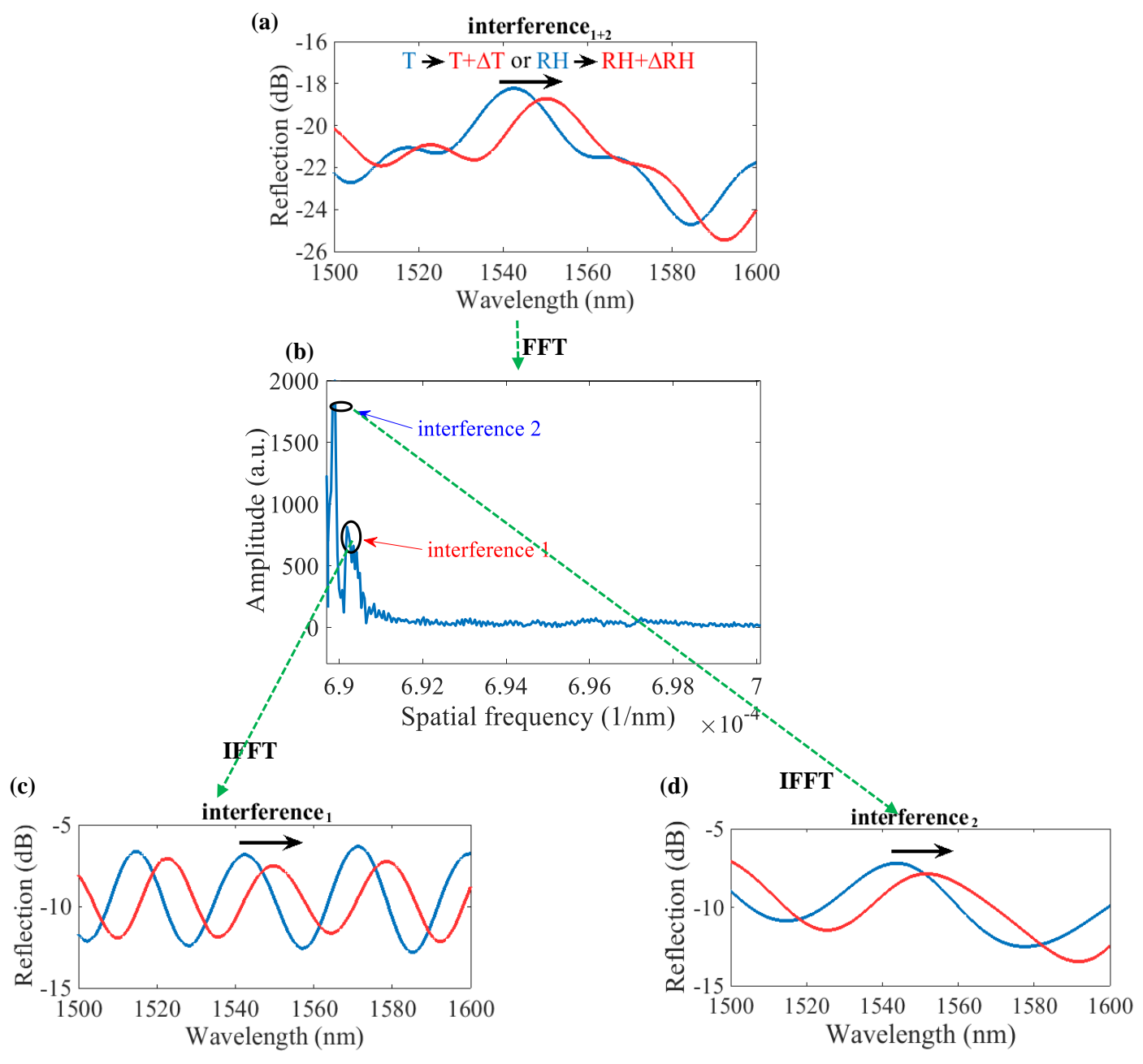

Figure 3. (a) Optical response of superimposed interference measured by the OSA; (b) superimposed spectra processed by FFT; separated optical spectra of (c) interference ${ }_{1}$ and

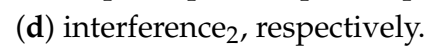


If there are changes in temperature $(\Delta T)$ and relative humidity $(\Delta R H)$, wavelength shifts both from sensor $_{1}\left(\Delta \lambda_{1}\right)$ and sensor $2\left(\Delta \lambda_{2}\right)$ can be respectively estimated using the simultaneous Equation $(1 \mathrm{a}, \mathrm{b})$. In mathematics, a system of linear equations is a collection of two or more linear equations involving the same set of variables. Thus, a solution with several variables to a linear system can be obtained in which all the equations are simultaneously satisfied. Thus, by solving the simultaneous Equation $(1 \mathrm{a}, \mathrm{b})$ of the sensing system, one can determine two variables $(\Delta T$ and $\Delta R H)$ at the same time.

$$
\begin{aligned}
& \frac{\Delta \lambda_{1}}{\lambda_{1}}=\frac{1}{d_{1}} \frac{\partial d_{1}}{\partial T} \Delta T+B \Delta R H=A \Delta T+B \Delta R H . \\
& \frac{\Delta \lambda_{2}}{\lambda_{2}}=\frac{1}{d_{2}} \frac{\partial d_{2}}{\partial T} \Delta T+D \Delta R H=C \Delta T+D \Delta R H .
\end{aligned}
$$

In Equation (1a,b), $A$ and $C$ as well as $B$ and $D$ are the corresponding $\mathrm{T}$ and RH sensitivity coefficients for sensor ${ }_{1}$ and sensor 2 , respectively. Here, $d_{1}$ and $d_{2}$ denote the lengths of the polymer cavity for sensor ${ }_{1}$ and sensor 2 , respectively. The coefficients $A, B, C$, and $D$ can be determined by measuring the individual spectral sensitivities of the two PFFIs in the DPFFI system with the variations in $T$ and $R H$. Then, using the matrix inversion method, variations in temperature $(\Delta T)$ and relative humidity $(\Delta R H)$ can be simultaneously obtained by measuring the wavelength shifts of $\operatorname{PFFI}_{1}\left(\Delta \lambda_{1}\right)$ and $\mathrm{PFFI}_{2}\left(\Delta \lambda_{2}\right)$, respectively. The relation of the above parameters according to the Equation (1) is described below:

$$
\left(\begin{array}{c}
\Delta T \\
\Delta R H
\end{array}\right)=\left(\begin{array}{cc}
A & B \\
C & D
\end{array}\right)^{-1}\left(\begin{array}{c}
\frac{\Delta \lambda_{1}}{\lambda_{1}} \\
\frac{\Delta \lambda_{2}}{\lambda_{2}}
\end{array}\right) .
$$

Equation (2) can be simplified as follows:

$$
\left(\begin{array}{c}
\Delta T \\
\Delta R H
\end{array}\right)=\left(\begin{array}{cc}
A^{\prime} & B^{\prime} \\
C^{\prime} & D^{\prime}
\end{array}\right)^{-1}\left(\begin{array}{c}
\Delta \lambda_{1} \\
\Delta \lambda_{2}
\end{array}\right)
$$

where $A^{\prime}, C^{\prime}, B^{\prime}$ and $D^{\prime}$ represent the normalized coefficients for the $\mathrm{T}$ and $\mathrm{RH}$ sensitivities of sensor 1 and sensor ${ }_{2}$, respectively. As a result, the sensitivity coefficients $A^{\prime}, C^{\prime}, B^{\prime}$, and $D^{\prime}$ in the sensing configuration need to be evaluated primarily to achieve the simultaneous measurement of RH and T.

\section{Experimental Results and Discussion}

In the experiment, the proposed DPFFI device was placed inside a temperature and humidity controlling chamber (THCC), as displayed in Figure 2, which was a closed space in which the T was increased from 20 to $50{ }^{\circ} \mathrm{C}$ and $\mathrm{RH}$ was fixed. The measured results are shown in Figure 4 . The spectral responses to $\mathrm{T}$ are displayed in Figure $4 \mathrm{a}, \mathrm{c}$ for sensor ${ }_{1}$ and sensor ${ }_{2}$, respectively. Based on the individual responses of interference ${ }_{1}$ and interference ${ }_{2}$ of sensor ${ }_{1}$ and sensor 2 , the $\mathrm{T}$ sensitivities of sensor $_{1}$ and sensor 2 to $\mathrm{T}$ are $+0.25376 \mathrm{~nm} /{ }^{\circ} \mathrm{C}$ and $+0.39551 \mathrm{~nm} /{ }^{\circ} \mathrm{C}$, respectively, plotted in Figure $4 \mathrm{~b}, \mathrm{~d}$. Again, the device was placed in a condition where RH was increased from 20 to $90 \%$ and $\mathrm{T}$ was fixed at $25{ }^{\circ} \mathrm{C}$. Figure 5 shows the RH sensitivities of sensor ${ }_{1}$ and sensor ${ }_{2}:+0.12538 \mathrm{~nm} / \% \mathrm{RH}$ and $+0.15807 \mathrm{~nm} / \% \mathrm{RH}$, respectively. These results demonstrate that the proposed sensor has a wavelength redshift response when either $\mathrm{T}$ or $\mathrm{RH}$ is increased. It can also be inferred that shorter cavity lengths have larger free spectral ranges (FSRs) with greater T and RH sensitivities. However, it could be that, because of the possible overlap of interference dips, the measurement range is limited by the length of the FSR. The amount of wavelength shifts approaching the FSR will lead to the overlap of interference signals, so the wavelength shifts cannot be well identified. To avoid an overlap of interference dips, appropriate ranges of $\mathrm{T}\left(20 \sim 50^{\circ} \mathrm{C}\right)$ and $\mathrm{RH}(20 \sim 90 \%)$ were considered in our study to obtain the $\mathrm{T}$ and $\mathrm{RH}$ sensitivity slopes and to demonstrate the proposed sensing method. 
(a)

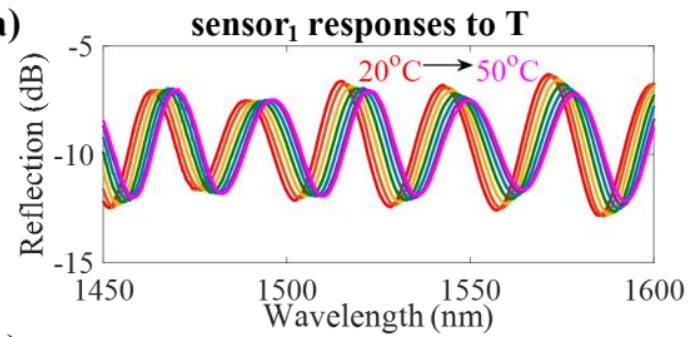

(c)

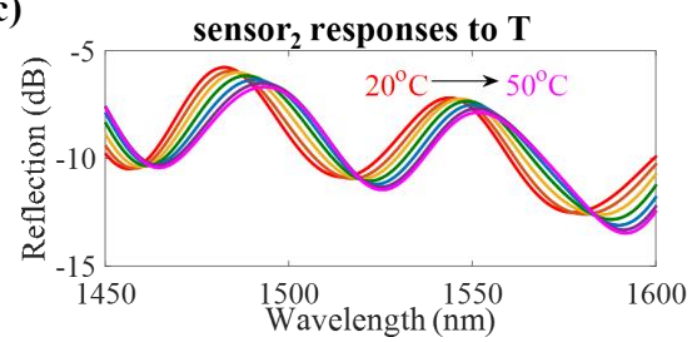

(b)

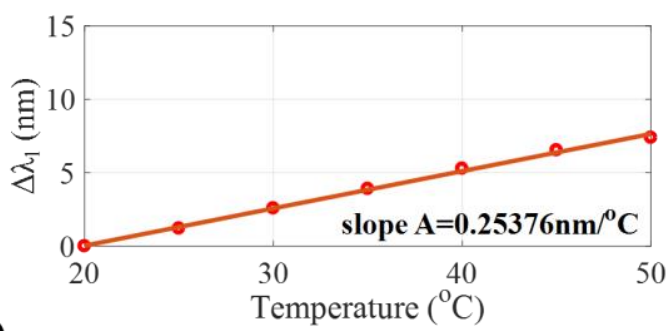

(d)

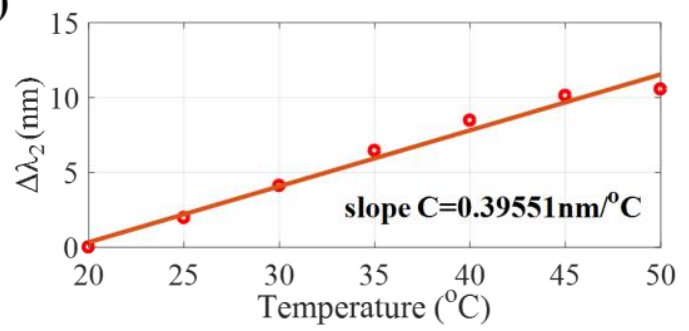

Figure 4. $(\mathrm{a}, \mathrm{c})$ Interference spectra of sensor ${ }_{1}$ and sensor 2 for different $\mathrm{T}$ values, respectively; $(\mathbf{b}, \mathbf{d})$ Corresponding $\mathrm{T}$ sensitivities for sensor ${ }_{1}$ and sensor 2 , respectively.

(a)

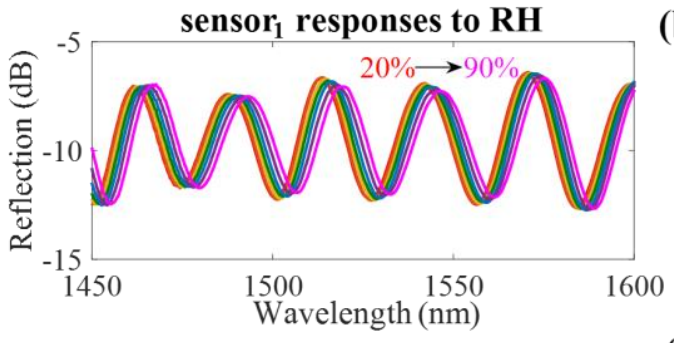

(c)

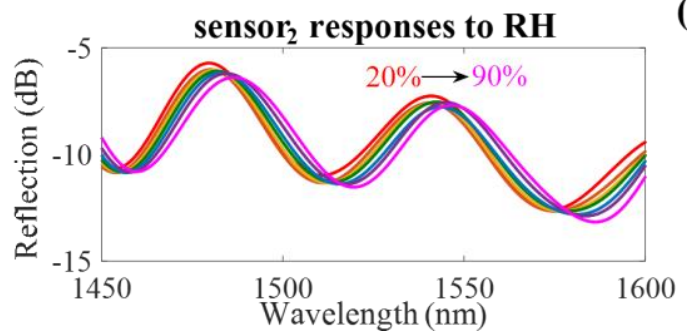

(b)

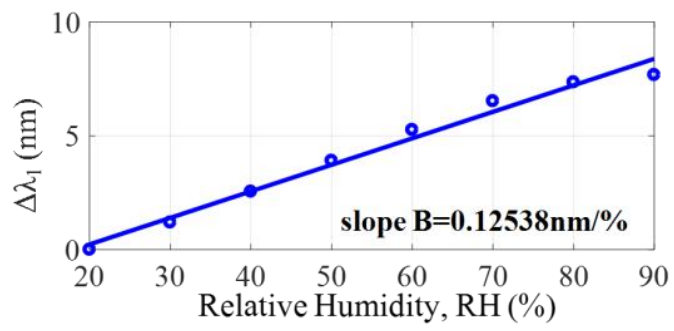

(d)

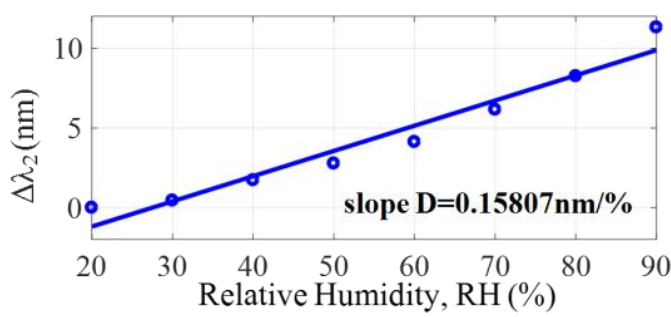

Figure 5. (a,c) Interference spectra of sensor ${ }_{1}$ and sensor 2 for different $\mathrm{RH}$ values, respectively; $(\mathbf{b}, \mathbf{d})$ Corresponding RH sensitivities for sensor ${ }_{1}$ and sensor 2 , respectively.

Based on the above experimental results, the normalized sensitivity parameters $A^{\prime}, B^{\prime}, C^{\prime}$, and $D^{\prime}$ are the T and RH sensitivity coefficients of sensor ${ }_{1}$ and sensor ${ }_{2}$, which are estimated to be $0.25376 \mathrm{~nm} /{ }^{\circ} \mathrm{C}, 0.12538 \mathrm{~nm} / \% \mathrm{RH}, 0.39551 \mathrm{~nm} /{ }^{\circ} \mathrm{C}$, and $0.15807 \mathrm{~nm} / \% \mathrm{RH}$, respectively. The matrix inversion method is then utilized to perform the simultaneous measurement of RH and T. The analytical simultaneous equation, Equation (3), can be expressed as Equation (4). The inverse matrix can then be used to simplify Equation (4), yielding Equation (5).

$$
\begin{gathered}
\left(\begin{array}{c}
\Delta T \\
\Delta R H
\end{array}\right)=\left(\begin{array}{cc}
A^{\prime} & B^{\prime} \\
C^{\prime} & D^{\prime}
\end{array}\right)^{-1}\left(\begin{array}{l}
\Delta \lambda_{1} \\
\Delta \lambda_{2}
\end{array}\right)=\left(\begin{array}{cc}
0.25376 & 0.12538 \\
0.39551 & 0.15807
\end{array}\right)^{-1}\left(\begin{array}{l}
\Delta \lambda_{1} \\
\Delta \lambda_{2}
\end{array}\right) . \\
\left(\begin{array}{c}
\Delta T \\
\Delta R H
\end{array}\right)=\frac{1}{-9.4914 \times 10^{-3}}\left(\begin{array}{cc}
0.25376 & -0.39551 \\
-0.12538 & 0.15807
\end{array}\right)\left(\begin{array}{c}
\Delta \lambda_{1} \\
\Delta \lambda_{2}
\end{array}\right) .
\end{gathered}
$$


To investigate the effectiveness of Equation (5) for the developed sensing configuration, $\mathrm{T}$ and $\mathrm{RH}$ were simultaneously varied from their reference values of ambient $\mathrm{T}_{0}=20^{\circ} \mathrm{C}$ and $\mathrm{RH}_{0}=20 \%$ to conditions shown in Figures 6 and 7. The optical spectra of the initial condition of $\mathrm{T}_{0}=20^{\circ} \mathrm{C}$ and $\mathrm{RH}_{0}=20 \%$ for sensor ${ }_{1}$ and sensor 2 were recorded at the beginning of the experimental tests (The blue line in Figure 7). The Figure 6 presents the simultaneous measurement of random $T$ and $\mathrm{RH}$ values under various levels. The blue circles represent the states of the T and RH levels in the temperature and humidity controlling chamber (THCC). The red triangles are the measured data evaluated by the proposed method. One can see that, in Figure 6, the simultaneous sensing of T and $\mathrm{RH}$ was accomplished. The average errors of the T and RH measurements in Figure 6 are about $\sim 1.46{ }^{\circ} \mathrm{C}$ and $\sim 1.48 \%$, respectively. We think that the errors are attributable to the measured deviations of the THCC machine and errors of numerical calculation. The results of three randomly selected cases-(a) $\mathrm{T}=50{ }^{\circ} \mathrm{C}, \mathrm{RH}=40 \%$, (b) $\mathrm{T}=40{ }^{\circ} \mathrm{C}, \mathrm{RH}=65 \%$, and (c) $\mathrm{T}=55^{\circ} \mathrm{C}, \mathrm{RH}=90 \%$ (marked in Figure 6) -whose measured spectral data and optical wavelength shifts are shown in Figure 7 and evaluated in Table 1, respectively.

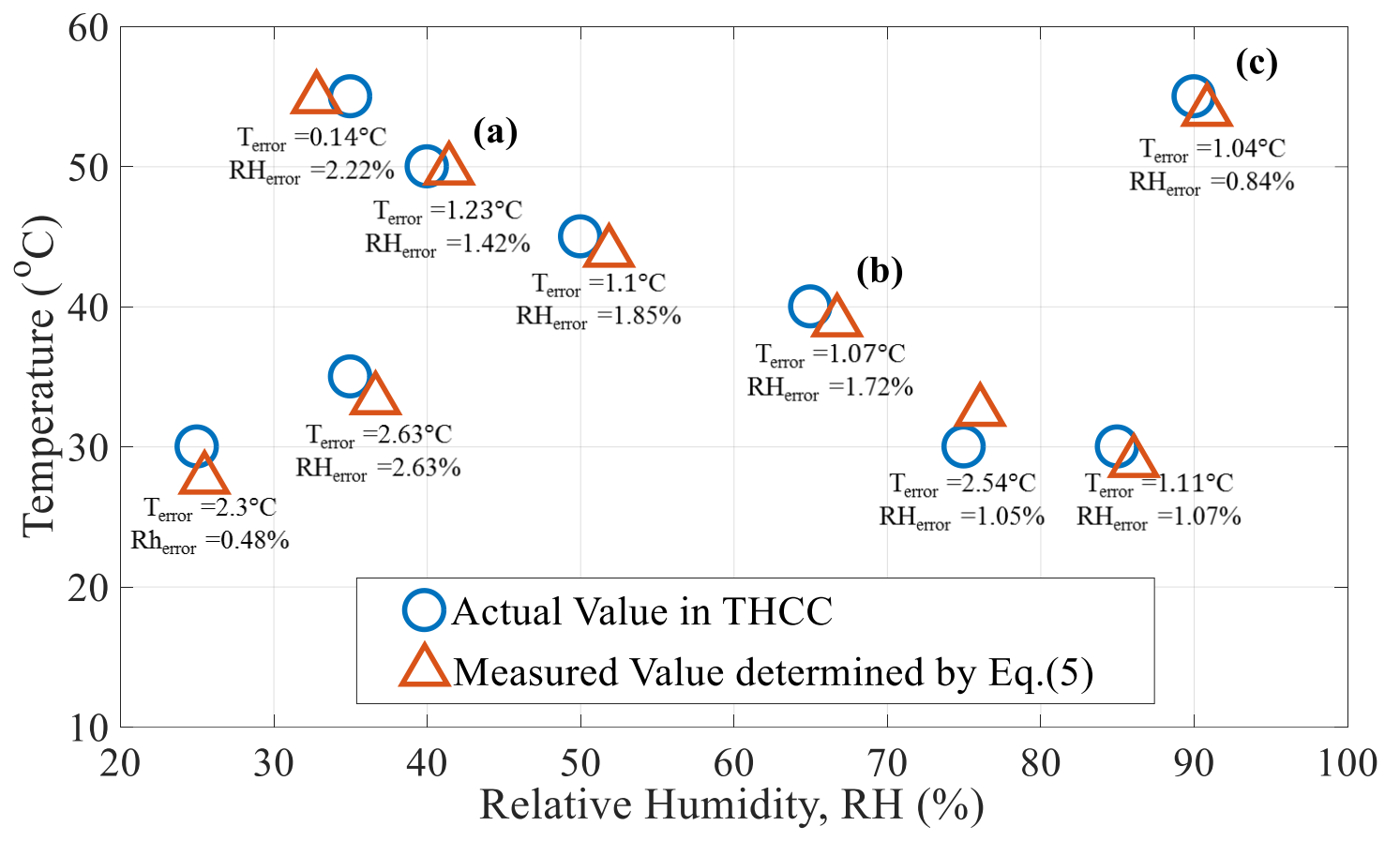

Figure 6. Sample data for measurement comparison.

In Condition (a) of Figure 7 as well as in Table 1, the measured wavelength shifts of sensor ${ }_{1}$ and sensor 2 are $\Delta \lambda_{1}=10.24 \mathrm{~nm}$ and $\Delta \lambda_{2}=15.16 \mathrm{~nm}$, respectively. Based on Equation (5), the changes in $\mathrm{T}$ and $\mathrm{RH}$ are readily obtained as $\Delta \mathrm{T}=+29.77^{\circ} \mathrm{C}$ and $\Delta \mathrm{RH}=+21.42 \%$, respectively. Thus, the measurements of $\mathrm{T}$ and $\mathrm{RH}$ in the Condition (a) are $\mathrm{T}_{\mathrm{m}}=20+29.77=49.77^{\circ} \mathrm{C}$ and $\mathrm{RH}_{\mathrm{m}}=20+21.42=41.42 \%$, respectively. The simultaneously measured values of $\mathrm{T}_{\mathrm{m}}$ and $\mathrm{RH}_{\mathrm{m}}$ are very similar to the actual values displayed in the THCC. The errors in T and $\mathrm{RH}$ are $0.23{ }^{\circ} \mathrm{C}$ and $1.42 \%$, respectively. The other two measurements of Conditions (b) and (c) with measured values of $\Delta \lambda_{1}=10.66 \mathrm{~nm}$ and $\Delta \lambda_{2}=14.87 \mathrm{~nm}$, and $\Delta \lambda_{1}=17.50 \mathrm{~nm}$ and $\Delta \lambda_{2}=24.63 \mathrm{~nm}$, used to determine $\mathrm{T}_{\mathrm{m}}$ and $\mathrm{RH}_{\mathrm{m}}$, are also shown in Table 1 and Figure 7. The above evaluation demonstrates that effectiveness of the proposed DPFFI sensor. 

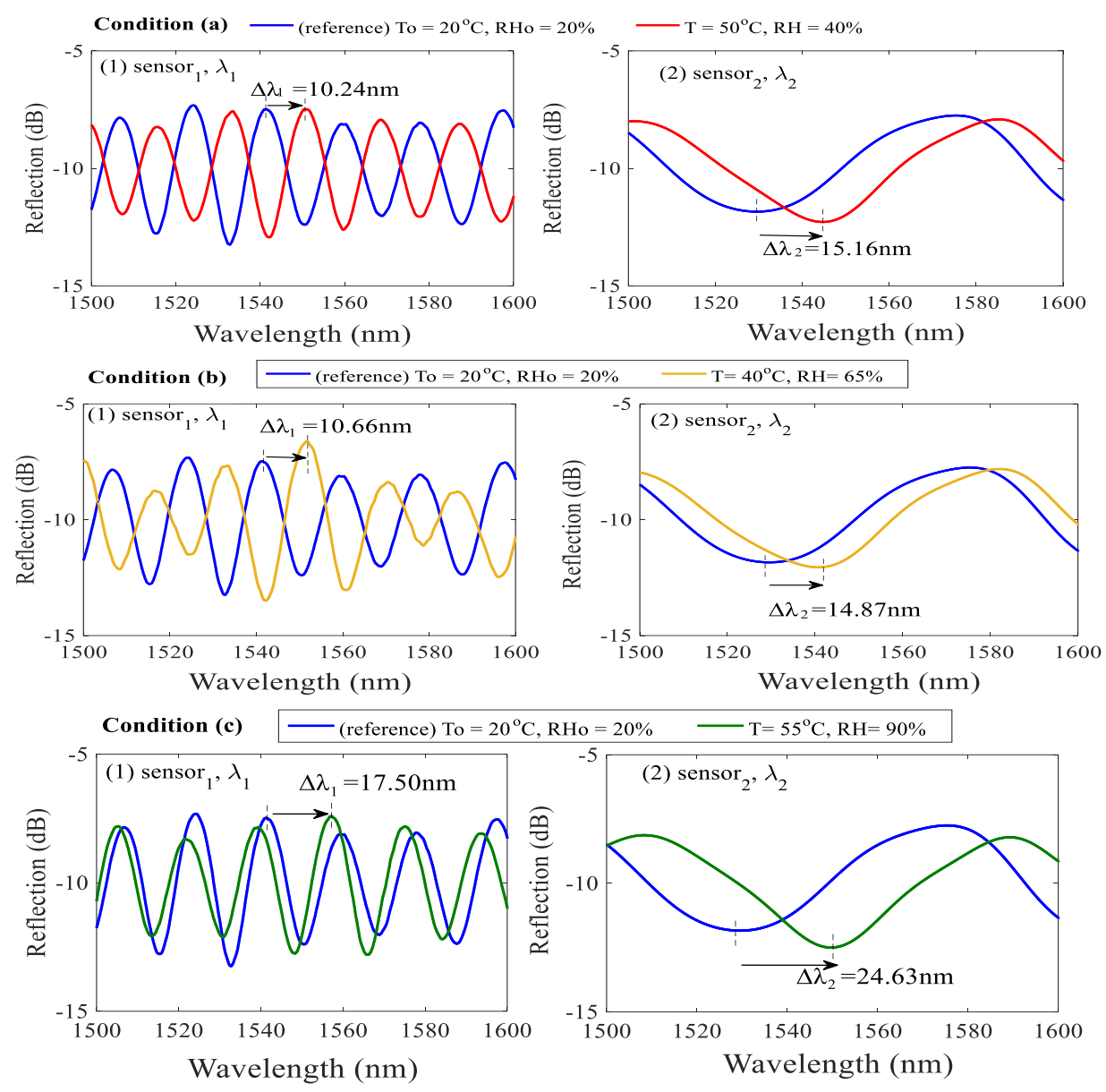

Figure 7. (1) and (2) are interference spectra shifts of sensor ${ }_{1}$ and sensor 2 , respectively, when RH and T change simultaneously in Conditions $(\mathbf{a}-\mathbf{c})$.

Table 1. Evaluating the simultaneous measurement of $\mathrm{T}$ and $\mathrm{RH}$ variations in different conditions of the proposed sensor.

\begin{tabular}{cccc}
\hline Pondition & (a) $\mathbf{T}=\mathbf{5 0}{ }^{\circ} \mathbf{C}, \mathbf{R H}=\mathbf{4 0} \%$ & (b) $\mathbf{T}=\mathbf{4 0}{ }^{\circ} \mathbf{C}, \mathbf{R H}=\mathbf{6 5} \%$ & (c) $\mathbf{T}=\mathbf{5 5}{ }^{\circ} \mathbf{C}, \mathbf{R H}=\mathbf{9 0} \%$ \\
\hline$\Delta \lambda_{1}(\mathrm{~nm})$ & +10.24 & +10.66 & +17.50 \\
$\Delta \lambda_{2}(\mathrm{~nm})$ & +15.16 & +14.87 & +24.63 \\
\hline Determined by & $\Delta \mathrm{T}=+29.77^{\circ} \mathrm{C}$ & $\Delta \mathrm{T}=+18.93{ }^{\circ} \mathrm{C}$ & $\Delta \mathrm{T}=+33.96{ }^{\circ} \mathrm{C}$ \\
Equation (5) & $\Delta \mathrm{RH}=+21.42 \%$ & $\Delta \mathrm{RH}=+46.72 \%$ & $\Delta \mathrm{RH}=+70.84 \%$ \\
\hline \multirow{2}{*}{ Measured $\mathrm{T}_{\mathrm{m}}$ and $\mathrm{RH}_{\mathrm{m}}$} & $\mathrm{T}_{\mathrm{m}}=\mathrm{T}_{0}+\Delta \mathrm{T}=49.77{ }^{\circ} \mathrm{C}$ & $\mathrm{T}_{\mathrm{m}}=\mathrm{T}_{0}+\Delta \mathrm{T}=38.93{ }^{\circ} \mathrm{C}$ & $\mathrm{T}_{\mathrm{m}}=\mathrm{T}_{0}+\Delta \mathrm{T}=53.96{ }^{\circ} \mathrm{C}$ \\
& $\mathrm{RH}_{\mathrm{m}}=\mathrm{RH}_{0}+\Delta \mathrm{RH}=41.42 \%$ & $\mathrm{RH}_{\mathrm{m}}=\mathrm{RH}_{0}+\Delta \mathrm{RH}=66.72 \%$ & $\mathrm{RH}_{\mathrm{m}}=\mathrm{RH}_{0}+\Delta \mathrm{RH}=90.84 \%$ \\
\hline $\mathrm{T}_{\text {error }}=\left|\mathrm{T}_{\mathrm{m}}-\mathrm{T}\right|$ & $\mathrm{T}_{\text {error }}=0.23{ }^{\circ} \mathrm{C}$ & $\mathrm{T}_{\text {error }}=1.07{ }^{\circ} \mathrm{C}$ & $\mathrm{T}_{\text {error }}=1.04{ }^{\circ} \mathrm{C}$ \\
$\mathrm{RH}_{\text {error }}=\left|\mathrm{RH}_{\mathrm{m}}-\mathrm{RH}\right|$ & $\mathrm{RH}_{\text {error }}=1.42 \%$ & $\mathrm{RH}_{\text {error }}=1.72 \%$ & $\mathrm{RH}_{\text {error }}=0.84 \%$ \\
\hline
\end{tabular}

\section{Conclusions}

We have developed a dual polymer fiber Fizeau interferometer (DPFFI) and used it to measure relative humidity (RH) and temperature (T) simultaneously using the fast Fourier transform (FFT) and a strategically mathematical matrix inversion relation. Since the proposed PFFI is highly sensitive to $\mathrm{T}$ and $\mathrm{RH}$, sensing configuration of the developed DPFFI strategically combined with two different PFFIs can individually identify the variations in $\mathrm{T}$ and $\mathrm{RH}$ to achieve the objective of simultaneous measurement. The sensing principle is based on the fact that both PFFIs exhibit diverse RH and T sensitivities, respectively. Comprehensive experimental tests have been accomplished. Consistent 
results show that, by using the proposed sensing scheme with a simple mathematical method, the proposed DPFFI can simultaneously and effectively measure $\mathrm{T}$ and $\mathrm{RH}$ with small errors in sensing ranges of $\mathrm{T}=20 \sim 50{ }^{\circ} \mathrm{C}$ and $\mathrm{RH}=20 \sim 90 \%$.

Acknowledgments: The authors would like to thank the Ministry of Science and Technology of Taiwan, MOST 105-2221-E-239-01 and MOST 106-2221-E-239-014 to support the research.

Author Contributions: This work was carried out in collaboration between all authors. The first author Chao-Tsung Ma proposed the sensing concept, verified the mathematical method, analyzed the data, revised and polished the final manuscript. Authors, Yu-Wei Chang and Yuan-Jie Yang, the undergraduate students in the Department of EOE, performed the experiments, managed figures and data. The corresonding author, Cheng-Ling Lee, designed the experiments, verified the results and wrote the first draft of the manuscript.

Conflicts of Interest: The authors declare no conflict of interest.

\section{References}

1. Arregui, F.J.; Matías, I.R.; Cooper, K.L.; Claus, R.O. Simultaneous measurement of humidity and temperature by combining a reflective intensity-based optical fiber sensor and a fiber Bragg grating. IEEE Sens. 2002, 2, 482-487. [CrossRef]

2. Mathew, J.; Semenova, Y.; Farrell, G. Fiber optic hybrid device for simultaneous measurement of humidity and temperature. IEEE Sens. J. 2013, 13, 1632-1636. [CrossRef]

3. Zhang, S.Q.; Dong, X.Y.; Li, T.; Chan, C.C.; Shum, P.P. Simultaneous measurement of relative humidity and temperature with PCF-MZI cascaded by fiber Bragg grating. Opt. Commun. 2013, 303, 42-45. [CrossRef]

4. Yan, G.F.; Liang, Y.H.; Lee, E.-H.; He, S. Novel Knob-integrated fiber Bragg grating sensor with polyvinyl alcohol coating for simultaneous relative humidity and temperature measurement. Opt. Express 2015, 23, 15624-15634. [CrossRef] [PubMed]

5. Massaroni, C.; Caponero, M.A.; D'Amato, R.; Lo Presti, D.; Schena, E. Fiber Bragg grating measuring system for simultaneous monitoring of temperature and humidity in mechanical ventilation. Sensors 2017, 17, 749. [CrossRef] [PubMed]

6. Yulianti, I.; Supa'at, A.S.M.; Idrus, S.M.; Anwar, M.R.S. Design of fiber Bragg grating-based Fabry-Perot sensor for simultaneous measurement of humidity and temperature. Optik 2013, 124, 3919-3923. [CrossRef]

7. Lee, C.L.; You, Y.W.; Dai, J.H.; Hsu, J.M.; Horng, J.S. Hygroscopic polymer microcavity fiber Fizeau interferometer incorporating a fiber Bragg grating for simultaneously sensing humidity and temperature. Sens. Actuators B Chem. 2016, 222, 339-346. [CrossRef]

8. Viegas, D.; Hernaez, M.; Goicoechea, J.; Santos, J.L.; Araújo, F.M.; Arregui, F.; Matias, I.R. Simultaneous measurement of humidity and temperature based on an $\mathrm{SiO}_{2}-\mathrm{Nanospheres} \mathrm{film} \mathrm{deposited} \mathrm{on} \mathrm{a} \mathrm{long-period}$ grating in-line with a fiber Bragg grating. IEEE Sens. J. 2010, 11, 162-166. [CrossRef]

9. Liu, H.; Liang, H.; Sun, M.; Ni, K.; Jin, Y. Simultaneous measurement of humidity and temperature based on a long-period fiber grating inscribed in fiber loop mirror. IEEE Sens. J. 2013, 14, 893-896. [CrossRef]

10. Urrutiaa, A.; Goicoecheaa, J.; Ricchiutib, A.L.; Barrerab, D.; Salesb, S.; Arreguia, F.J. Simultaneous measurement of humidity and temperature based on a partially coated optical fiber long period grating. Sens. Actuators B Chem. 2016, 227, 135-141. [CrossRef]

11. Yang, M.; Xie, W.; Dai, Y.; Lee, D.; Dai, J.; Zhang, Y.; Zhuang, Z. Dielectric multilayer-based fiber optic sensor enabling simultaneous measurement of humidity and temperature. Opt. Express 2014, 22, 11892-11899. [CrossRef] [PubMed]

12. Pevec, S.; Donlagic, D. Miniature all-silica fiber-optic sensor for simultaneous measurement of relative humidity and temperature. Opt. Lett. 2015, 40, 5646-5649. [CrossRef] [PubMed]

13. Sun, H.; Zhang, X.L.; Yuan, L.T.; Zhou, L.B.; Qiao, X.G.; Hu, M.L. An Optical Fiber Fabry-Perot Interferometer Sensor for Simultaneous Measurement of Relative Humidity and Temperature. IEEE Sens. J. 2015, 15, 2891-2897. [CrossRef]

(C) 2017 by the authors. Licensee MDPI, Basel, Switzerland. This article is an open access article distributed under the terms and conditions of the Creative Commons Attribution (CC BY) license (http:/ / creativecommons.org/licenses/by/4.0/). 